\title{
Tensile properties of amniotic membrane
}

\author{
K. Tanaka, T. Nagayama, T. Katayama \& N. Koizumi \\ Department of Biomedical Engineering, Doshisha University, Japan
}

\begin{abstract}
The amniotic membrane, which is the innermost layer of the placental membrane, is a thin and transparent tissue. Rejection rarely occurs in the amniotic membrane because it is free from vascular components. Therefore, the amniotic membrane has attracted attention as a surgical biomaterial for regenerative medicine in various medical fields. However, evaluation of mechanical properties for the amniotic membrane is not standardized because of the difficulty in handling tiny amniotic membrane materials.

In this study, to propose the most appropriate tensile testing method for the amniotic membrane, the influence of specimen shapes and testing jig types on the mechanical properties of the amniotic membrane is examined. Moreover, for the strain measurement of the thin film like amniotic membrane, due to the low rigidity of the specimen, it is necessary to measure the strain by a non-contact measurement method. A non-contact optical 3D deformation measuring system, which can measure strain by the image correlation method using the dotted pattern of specimen's surface, is applied for the tensile testing of the amniotic membrane. The dumbbell specimen and grip jig are shown to be suitable for tensile testing of the amniotic membrane. The tensile strength of the amniotic membrane was $6.80 \pm 0.22 \mathrm{MPa}$. For strain measurement by using a non-contact optical 3D deformation measuring system, the organic solvent spray was not suitable because the ink-film was formed on the surface of the amniotic membrane. On the other hand, spray mixed with calcium carbonate and hydrophilic ink was appropriate to apply a dotted pattern on the surface of the amniotic membrane.
\end{abstract}

Keywords: amniotic membrane, tensile test, non-contact optical 3D deformation measuring system. 


\section{Introduction}

The amniotic membrane, which is the innermost layer of the placental membrane, is a thin and transparent tissue. Rejection rarely occurs in the amniotic membrane because it is free from vascular components [1]. Therefore, the amniotic membrane has attracted attention as a surgical biomaterial for regenerative medicine in various medical fields [2-5]. The regenerative medicine is a medical treatment that regenerates tissues damaged by disorders and external injuries using cultivated cells and tissues in vitro. The amniotic membrane is expected to be used for the substrate of the skin, the eardrum, and the cornea. Particular attention has been focused on the amniotic membrane in the ophthalmologic field. It has been reported that the amniotic membrane has various kinds of biological characteristics, such as an anti-inflammatory effect, anti-fibroblastic activity, anti-microbial and anti-angiogeneic properties, which are suited for use in ocular surface reconstruction [6]. However, some problems, such as the deficiency of appropriate sterilization and difficulties in transport and storage, still remain. Human amniotic membrane is usually obtained instantaneously from a caesarean section and cryopreserved at $-80{ }^{\circ} \mathrm{C}$ under sterile conditions. In view of the attention focused on various pathogenic organisms, however, a proper sterilization method of the amniotic membrane should be developed. Currently the freeze-dried amniotic membrane that can be sterilized has been developed. With the amniotic membrane deriving from human bodies, it is impossible to obtain completely sterilized materials without any unknown existence of virus. Therefore, a material similar to amniotic membrane that is high in strength and low in rigidity is expected to be developed.

Currently, with the difficulty in handling tiny amniotic membrane materials, even the basic mechanical properties of the amniotic membrane are not clearly revealed. Oyen's research group [7] and Nakamura's research group [6] have already reported the mechanical properties of the amniotic membrane. In their researches, there still exist various issues to be overcome in the evaluation of the mechanical properties of amniotic membrane. For instance, there is a problem that tensile strength cannot be measured accurately due to the stress concentration at the chucking point for rectangular specimens. Moreover, as the amniotic membrane is gripped directly by a clip for installation to the testing machine, the amniotic membrane will easily come off from a gripping jig during the tensile test.

In this study, to propose the most appropriate tensile testing method for the amniotic membrane, the influence of specimen shapes and testing jig types on the mechanical properties of the amniotic membrane is examined. Moreover, for the strain measurement of the thin film like amniotic membrane, due to the low rigidity of the specimen, it is necessary to measure the strain by a non-contact measurement method. A non-contact optical 3D deformation measuring system, which can measure strain by the image correlation method using the dotted pattern of the specimen's surface, is applied for the tensile testing of the amniotic membrane. To optimize the patterning method for the strain measurement, 
different kinds of ink were applied to the specimen surface of the amniotic membrane.

\section{Materials and experimental procedure}

\subsection{Material and specimens}

Specimens of human amniotic membrane were obtained from the Supporting Organization for Regenerative Medicine. The study protocol was approved by the university ethical committee. Specimens were preserved in the freezer at $-80{ }^{\circ} \mathrm{C}$ in asepsis, and the chorion membrane was peeled off from the amniotic membrane. The amniotic membrane was defrosted at room temperature and washed by using Phosphate Buffered Saline (PBS) before being examined. Two types of specimen shapes, a dumbbell shape and rectangular shape, were used. The dumbbell size of the specimen was $5 \mathrm{~mm}$ in width, $20 \mathrm{~mm}$ in length, $1.5 \mathrm{~mm}$ in the centre part width, and $3 \mathrm{~mm}$ in the gauge length as shown in Fig.1(a), and

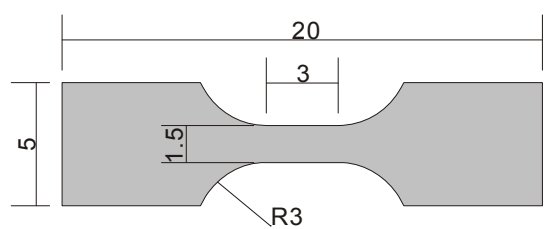

(a) Dumbbell shape

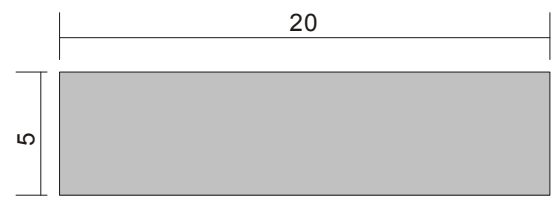

(b) Rectangular shape

Figure 1: $\quad$ Shape and dimensions.

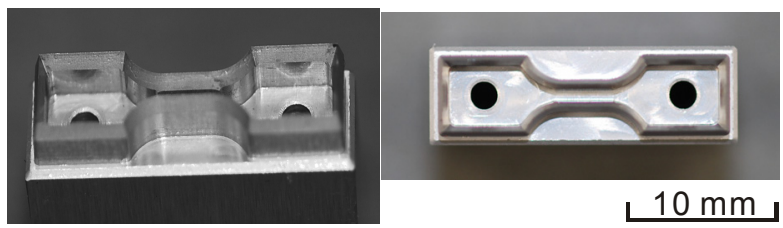

Figure 2: $\quad$ Cutter shape of cutting die.

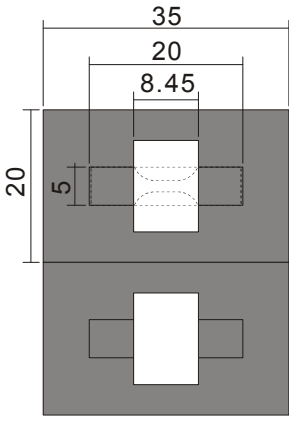

(a) Tab for grip jig.

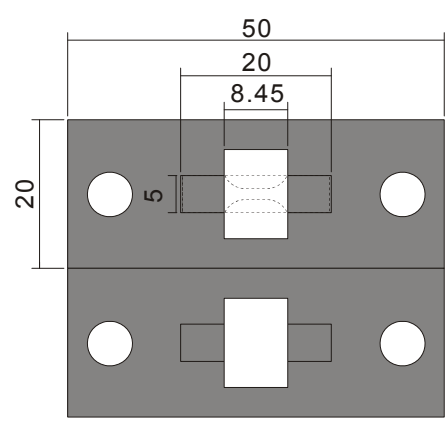

(b) Tab for pin jig.

Figure 3: $\quad$ Tab made of polyester thin film. 

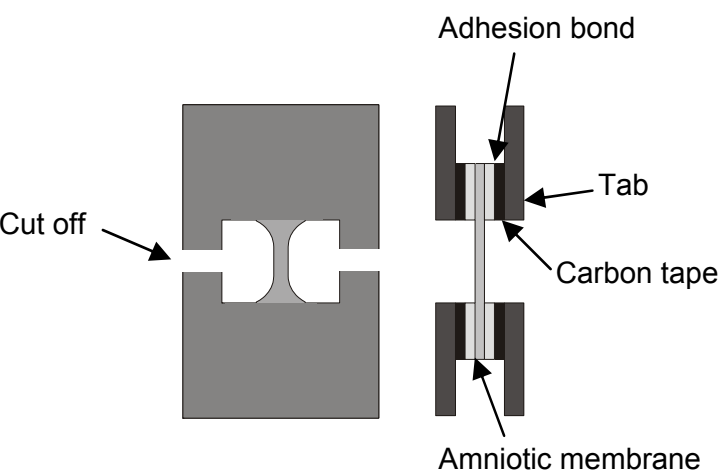

Figure 4: The fixation method of the amniotic membrane.

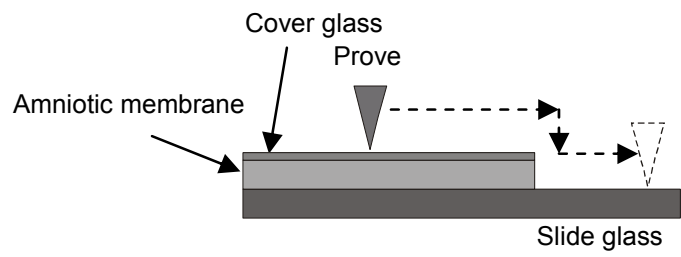

Figure 5: $\quad$ Measurement method for amniotic membrane thickness.

the stress concentration factor of $\mathrm{R}$ is 1.2. A dumbbell shaped specimen was made by pressing a cutting die, as shown in fig. 2 , into the amniotic membrane. The size of the rectangular specimen was adjusted to $5 \times 20 \mathrm{~mm}$ as shown in Fig. 1(b). The rectangular shaped specimen was made by pressing a cutting die with two blades at $5 \mathrm{~mm}$ apart into the amniotic membrane. The tab was made by polyester thin film as shown in Fig.3. The amniotic membrane has been griped and fixed in place to a tab by using carbon tape and adhesive glue (cyanoacrylate system) as shown in fig. 4 .

\subsection{Measurement of amniotic membrane thickness}

Amniotic membrane thickness was measured by using the surface profiler (KLA-Tencor, ALPHA STEP IQ). The amniotic membrane, being a soft material that contains water, was placed on the slide glass and topped by a cover glass as shown in fig.5. Afterwards, the surface of the cover glass was traced and measured with a probe of the contact type. The thickness of the amniotic membrane was calculated by measuring the total thickness of the cover glass together with the amniotic membrane, and then subtracting the thickness of the cover glass from this value. 


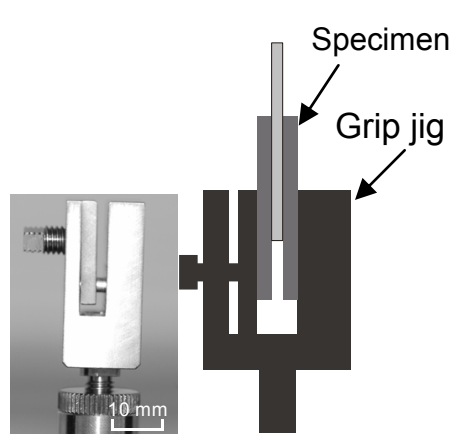

(a) Grip jig.

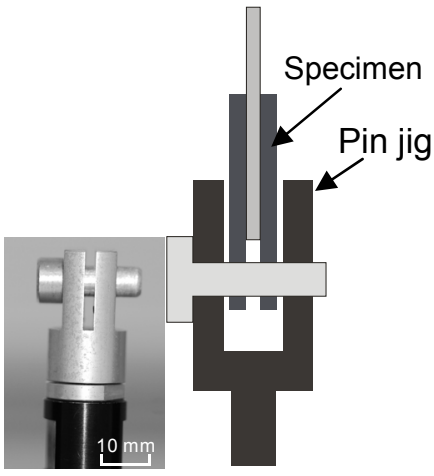

(b) Pin jig.

Figure 6: Gripping method.

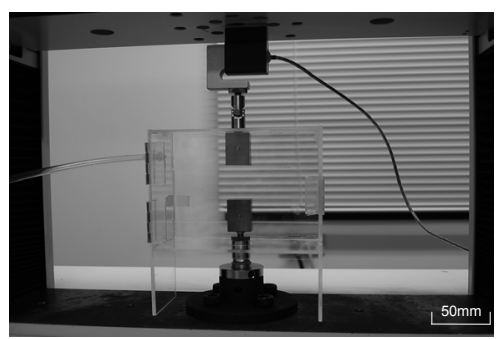

Figure 7: Test circumstance.

\subsection{Tensile test}

A universal mechanical testing machine (EZ-graph, SHIMADZU Co) with 100 $\mathrm{N}$ load cell is used for the tensile test. As shown in Fig.6, two types of jigs (grip type and pin type) were used for the installation of the amniotic membrane onto the testing machine. Both ends of the tab were cut, as shown in Fig.4, after the specimen was fixed to the testing machine. The load was applied to only the amniotic membrane, and the tensile test began. The crosshead speed was 0.05 $\mathrm{mm} / \mathrm{sec}$. The entire specimen was covered in an acrylic container, and its inside was filled with steam, as shown in Fig.7, so that the amniotic membrane would not dry up, and $100 \%$ humidity was maintained throughout the examination. For both dumbbell and rectangular specimens, three samples of each were used for pin and grip jig testing.

\subsection{Strain measurement}

The strain of the amniotic membrane was measured by using the non-contact optical 3D deformation measuring system (ARAMIS, GOM mbH). ARAMIS is a measurement apparatus that measures the strain according to the image correlation method by having a sprayed pattern on specimens. Generally, for 
applying pattern on the specimens, an organic solvent spray is used. In this study spray mixed with organic solvent was compared with spray mixed with calcium carbonate and low hydrophile ink in order to examine the effects of different organic solvents on the amniotic membrane.

\section{Results and discussion}

\subsection{Thickness measurement}

The thickness of one amniotic membrane specimen measured every two minutes is shown in fig.8. The result shows that thickness decreases over time and the decreasing rate slows down gradually. It can be considered that excess water of the amniotic membrane vaporized during the first phase (the decreasing slope is high), and water inside the amniotic membrane vaporized during the next phase (the decreasing slope is low). Therefore, the point that changed the inclination within this study has been defined as the thickness of the amniotic membrane that contained moisture, and resulted $50 \pm 7.7 \mu \mathrm{m}$. From reported transmission electron microscope (TEM) observation with a thickness measured at $50 \mu \mathrm{m}$ [6], it is clear that film thickness measurement could be carried out by using the surface profiler.

\subsection{Specimen shape and gripping jig}

Figs. 9 and 10 show the rectangular specimen and dumbbell specimen before and after its fracture. As the shown in Fig.9, the rectangular specimen had cracks appearing at the gripping point, the end of the gauge. The dumbbell specimen was ruptured in the centre. For the dumbbell specimen, the amniotic membrane of deformation size is large, and the centre parallel part of the dumbbell is fractured instead of the stress concentration point R. Fig.12 shows the

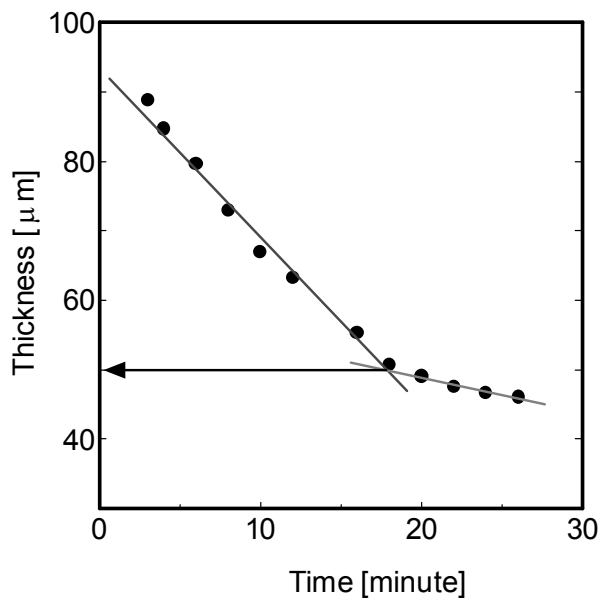

Figure 8: $\quad$ Measurement of thickness. 
load-displacement curve of dumbbell specimen for pin jig and grip jig. As a result, when load was over $0.4 \mathrm{~N}$, slipping occurred for tests done by the pin jig and tensile strength could not be measured; however, it could be measured by the grip jig. According to the above result, it is considered that the appropriate method for the dumbbell specimen would be by using the grip jig. In addition, amniotic membrane is a material which has nonlinear behaviour. In this study, we defined the maximum nominal stress on the tensile strength dividing the tensile load by the original centre parallel part and it was $6.80 \pm 0.22 \mathrm{MPa}$.

\subsection{Effect of paint on strain measurement}

Fig.12 shows the result of observing the surface of an amniotic membrane during the tensile test for specimen with an organic solvent spray and for specimen with a mixed spray containing calcium carbonate and low hydrophile ink. Moreover, the SEM image observed from the cross section surface of each sprayed amniotic membrane is shown in fig.13. Fig.12(a) shows clearly that cracks appeared on the ink film sprayed in the organic solvent. Such ink sprayed film breaks while the amniotic membrane stretched widely. On the other hand, as shown in fig.12(b) the mixture of calcium carbonate and ink stretches in accordingly to the amniotic membrane. As shown in fig.13, while the spray of organic solvent

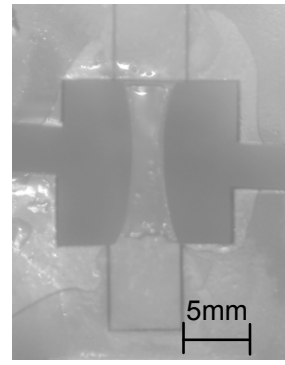

(a) Under tensile load.

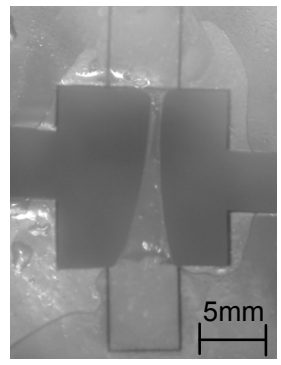

(b) Crack growth at chuck.

Figure 9: The tensile test for the rectangular specimen.

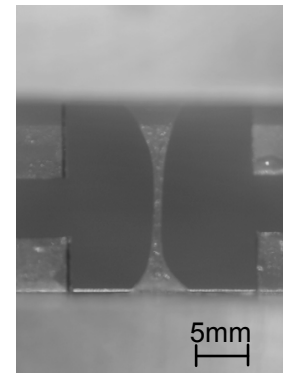

(a) Under tensile load.

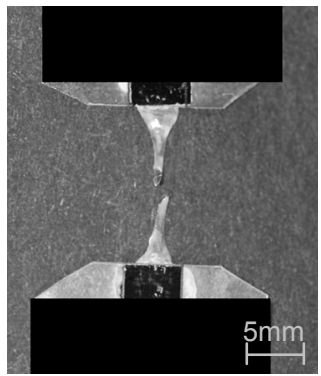

(b) After tensile test.

Figure 10: Tensile test for the dumbbell specimen. 


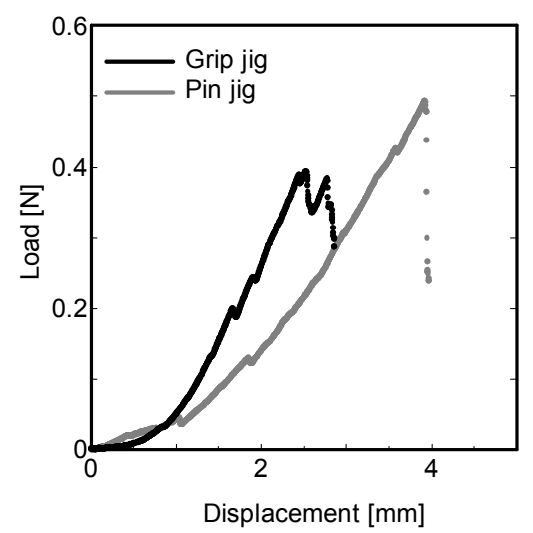

Figure 11: Load-displacement curves.

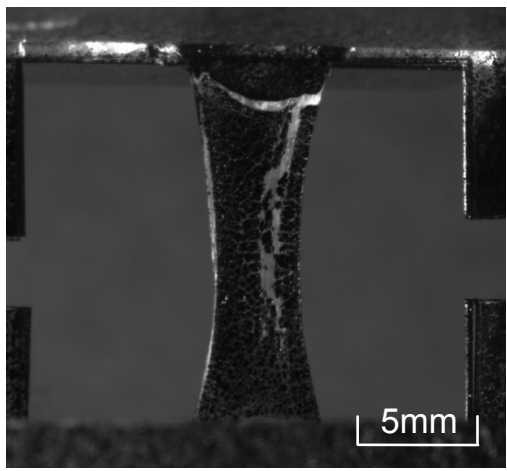

(a) Amniotic membrane with the organic solvent spray.

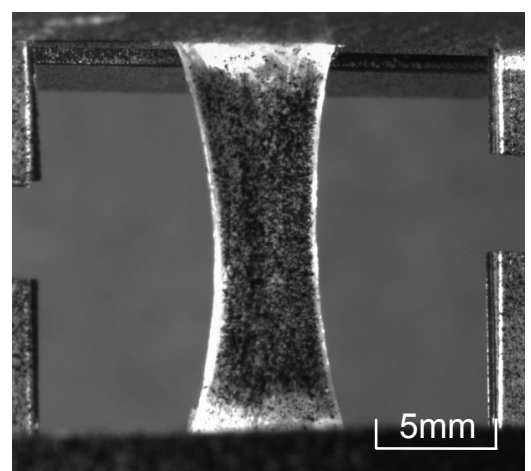

(b) Amniotic membrane with a spray containing calcium carbonate and low hydrophile ink.

Figure 12: The surface of the amniotic membrane. 


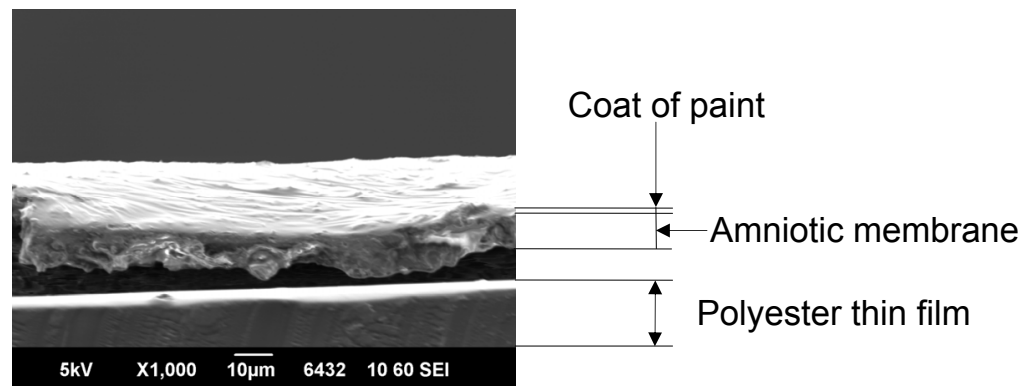

(a) The amniotic membrane with an organic solvent spray.

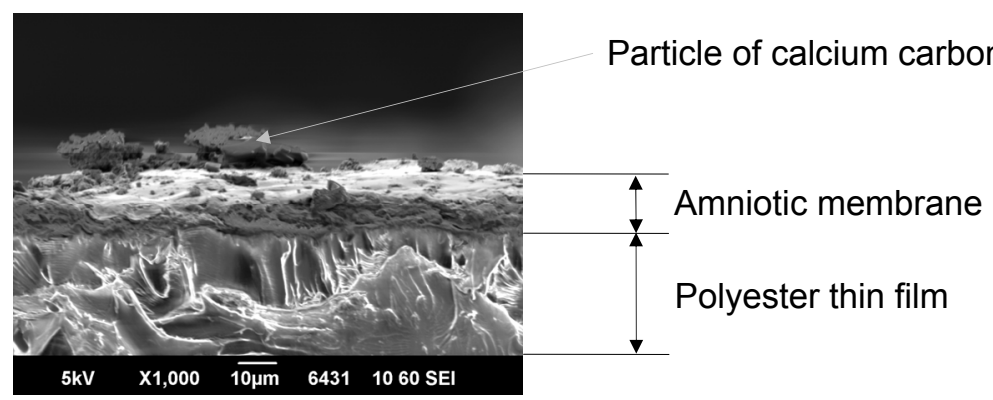

(b) The amniotic membrane with a mixed spray containing calcium carbonate and low hydrophile ink.

Figure 13: SEM observation of the cross section.

formed a thin film on the amniotic membrane (Fig.13(a)), the spray mixed with calcium carbonate and ink did not form a thin film, but rather simply had particles sitting on the amniotic membrane (Fig.13(b)).

\section{Conclusion}

In this study, the mechanical properties of the amniotic membrane for tensile testing are examined. The investigation yielded the following conclusions.

1. The dumbbell specimen and grip jig are suitable for tensile test of the amniotic membrane.

2. The tensile strength of the amniotic membrane is $6.80 \pm 0.22 \mathrm{MPa}$.

3. Spray mixed with calcium carbonate and hydrophilic ink was suitable as paints for the non-contact optical 3D deformation measuring system.

\section{Acknowledgement}

This research was partially supported by the Ministry of Education, Culture, Sports, Science and Technology; Grant-in-Aid for Young Scientists (B), 21760087, 2009 


\section{References}

[1] Noriko Koizumi et al., Cultivated corneal epithelial stem cell transplantation in ocular surface disorders, Ophthalmology 108(9), pp.1569-1574, 2001.

[2] Noriko Koizumi et al., Cultivation of corneal epithelial cells on intact and denuded human amniotic membrane, Invest Ophthalmol Vis Sci 41(9), pp.2506-2513, 2000.

[3] Noriko Koizumi et al., Ocular surface reconstruction, amniotic membrane, and cultivated epithelial cells from the limbus, $\mathrm{Br} J$ Ophthalmol 87, pp.1437-1439, 2003.

[4] ArBlast Co., Ltd., et al., Sheet-like Composition, PCT JP2006 314245 , 2006.

[5] Takahiro Nakamura et al., The use of trehalose-treated freeze-dried amniotic membrane for ocular surface reconstruction, Biomaterials 29, pp.37293737, 2008.

[6] Takahiro Nakamura et al., Sterilized, Freeze-Dried Amniotic Membrane: A Useful Substrate for Ocular Surface Reconstruction, Investigative Ophthalmology \& Visual Science, vol.45, No.1, pp.93-99, 2004.

[7] Michelle L. Oyen et al., Uniaxial and biaxial mechanical behavior of human amnion, J. Mater. Res., vol.20, No.11, pp.2902-2909, 2005. 